\title{
Exploring the X-ray emission properties of the supernova remnant G67.7+1.8 and its central X-ray sources
}

\author{
C. Y. Hui and W. Becker
}

\begin{abstract}
Max-Planck Institut für Extraterrestrische Physik, Giessenbachstrasse 1, 85741 Garching bei München, Germany e-mail: dhui@mpe.mpg.de
\end{abstract}

Received 12 August 2008 / Accepted 4 December 2008

ABSTRACT

\begin{abstract}
We have studied the supernova remnant G67.7+1.8 with the Chandra X-ray observatory. The remnant's X-ray morphology correlates well with the double-arc structure seen at radio wavelength. The X-ray spectra of the northern and southern rim of G67.7+1.8 exhibit emission line features of highly ionized metals, which suggests that most of the observed X-rays originate in a thermal plasma. We find magnesium, silicon, and sulphur overabundant relative to the solar values. Gaussian emission lines at $\sim 4 \mathrm{keV}$ and $\sim 7 \mathrm{keV}$ are detected. The $\sim 4 \mathrm{keV}$ line is consistent with $\mathrm{K}$-emission lines from ${ }^{44} \mathrm{Ca}$ and/or ${ }^{44} \mathrm{Sc}$ whereas the $\sim 7 \mathrm{keV}$ line feature may arise from unresolved Fe-K lines. Chandra's sub-arcsecond angular resolution allowed us to detect four faint point sources located within $\sim 1.5$ arcmin of the geometrical remnant center. Among these objects, CXOU195424.75+312824.9 and CXOU195429.82+312834.1 do not have optical counterparts, leaving them as candidates for a possible compact stellar remnant.
\end{abstract}

Key words. X-rays: stars - stars: neutron - stars: supernovae: individual: G67.7+1.8 - ISM: supernova remnants

\section{Introduction}

Thanks to the progress made in high-energy astrophysics in recent years other manifestations of neutron stars besides accretion- and rotation-powered pulsars have been found. Among the various different categories known today, central compact objects (CCOs) are the most enigmatic ones. These sources show up as relatively faint $\mathrm{X}$-ray point sources located close to the expansion center of their host supernova remnants (SNRs), strongly suggesting that they are indeed the compact remnants formed in the supernovae. Despite their apparently young age, the emission properties of CCOs are found to be very different from those observed in young rotation-powered pulsars. In the latter the X-ray emission is generally dominated by magnetospheric emission whereas the X-ray spectra of CCOs can be described well by composite blackbody models of temperatures $\left(T_{1}, T_{2}\right) \sim(3-7) \times 10^{6} \mathrm{~K}$ and radii $\left(R_{1}, R_{2}\right) \sim 0.3-$ $3 \mathrm{~km}$ of the projected blackbody emitting areas that are much smaller than the canonical neutron star radius (see Becker 2008, for a review). In contrast to the young Crab- and Vela-like pulsars, no plerionic emission has been found around CCOs (see Hui \& Becker 2006, and references therein).

The temporal emission behavior of the known CCOs is another puzzle. So far, X-ray pulses have been found from three of the seven known sources. The detected periodic signals span a wide range from $P \sim 0.1 \mathrm{~s}$ for the compact object in SNR Kes 79 (Halpern et al. 2007) to $P \sim 6.27 \mathrm{~h}$ in the case of the central point source in RCW 103 (De Luca et al. 2006). Different solutions have been proposed to explain their rotational dynamics. There is, however, no consensus so far on whether these objects e.g. were born with a slow spin and a weak magnetic field (Halpern et al. 2007) or at the other extreme that resembles the magnetars with a very short initial spin period and a very strong magnetic field (Li 2007).
Currently, only seven SNRs have been confirmed as hosting a CCO (cf. Tables 6.3 and 6.4 in Becker 2008). For a better understanding of their nature, the sample of CCOs needs to be increased. We therefore inspected known supernova remnants for centrally peaked X-ray emission by reanalyzing the ROSAT all-sky survey (RASS) data. G67.7+1.8 showed up in RASS data to have centrally bright X-ray emission that could potentially be associated with a compact source. A faint radio source seen in NRAO/VLA Sky Survey (NVSS) data (Condon et al. 1998) close to the geometrical center of the remnant correlates well with the central X-ray emission (cf. Fig. 1). The latter finding raised the question of whether this emission may eventually come from a young powerful pulsar and/or its synchrotron nebula. However, searching for a radio pulsar in G67.7+1.8 did not yield any detection. This non-detection placed an upper limit of $800 \mu \mathrm{Jy}$ on pulsed radio emission at $600 \mathrm{MHz}$ within $\mathrm{G} 67.7+1.8$ (Lorimer et al. 1998). Although this limit is not too constraining, it may suggest that the central emission could probably be associated with a $\mathrm{CCO}$ rather than with a young powerful rotationpowered pulsar.

G67.7+1.8 was first discovered in a Galactic plane survey at $327 \mathrm{MHz}$ by Taylor et al. (1992), using the Westerbork Synthesis Radio Telescope. The radio image has shown that G67.7+1.8 has a double arc structure. The angular diameter is $\sim 9^{\prime}$. Its distance is quite uncertain. The only distance estimate available comes from the $\Sigma-D$ relation, which puts the remnant at $\sim 17 \mathrm{kpc}$ (Case \& Bhattacharya 1998). However, optical filaments associated with the northern radio arc of G67.7+1.8 were found by Mavromatakis et al. (2001) and indicated that the remnants distance might actually be smaller. In the same work Mavromatakis et al. (2001) concludes that the energy released during the supernova explosion was significantly less than the canonical value of $10^{51}$ ergs.

$\mathrm{X}$-ray emission in the direction towards G67.7+1.8 was first observed in the ROSAT all-sky survey 


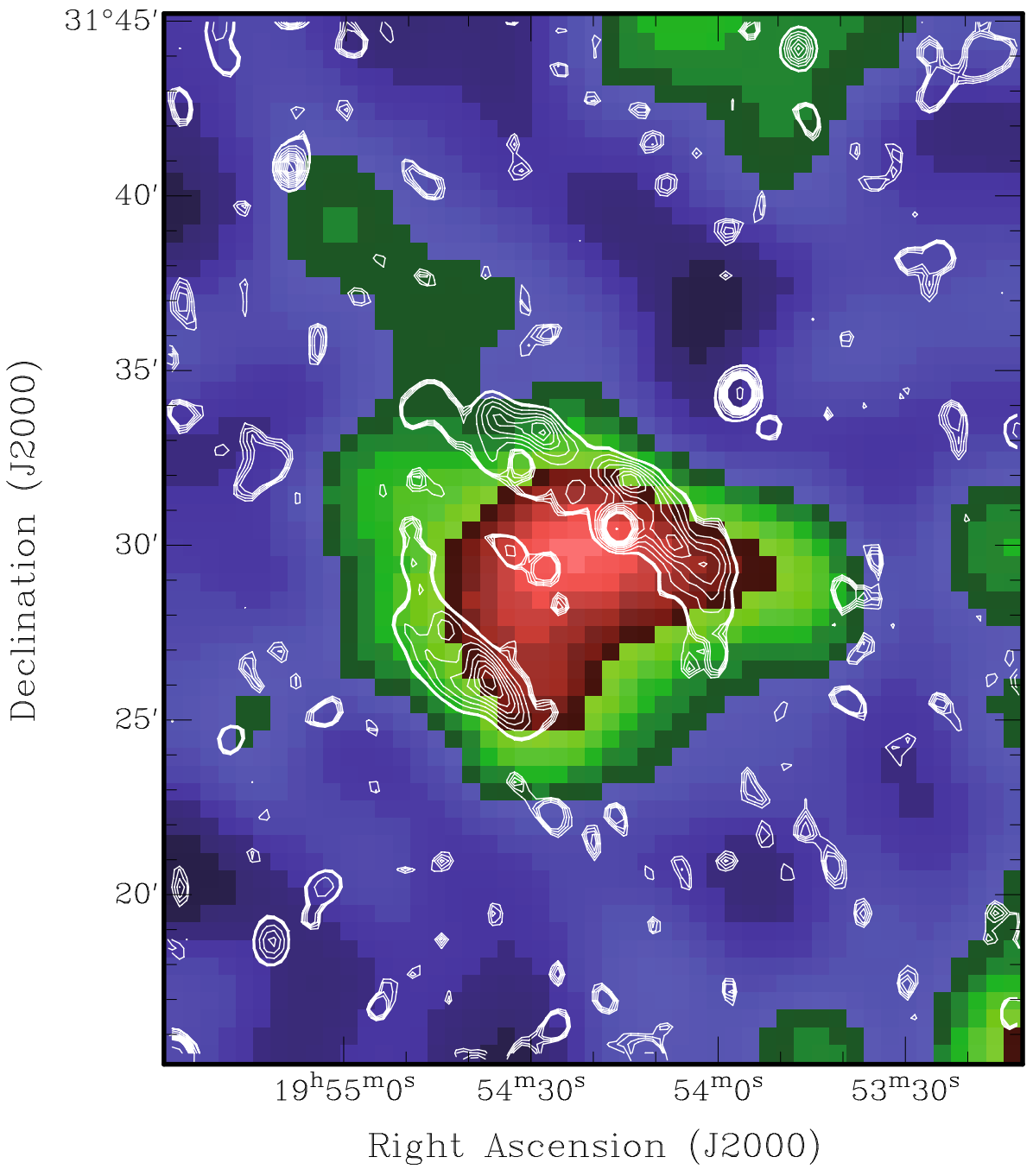

Fig. 1. ROSAT PSPC RASS image of G67.7+1.8 (1.0-2.0 keV) overlaid with contours from the NVSS survey data. The remnant appears center-filled in this data and has a bilateral radio arc-like structure. The peak of the central X-ray emission is found to be roughly matching the geometric center of the remnant. Faint radio sources are seen in the NVSS data near to the remnant center.
(Mavromatakis et al. 2001). Fitting the data with a thermal bremsstrahlung model indicated a plasma temperature in the range of $\sim 0.2-0.3 \mathrm{keV}$.

In this paper we present a detailed X-ray study of G67.7+1.8 and investigate the nature of its centrally peaked emission using Chandra ACIS-I observations. Its superior angular resolution enables us to perform a spectro-imaging study of the supernova remnant and to search for compact sources and/or a pulsar-wind nebula in its central part.

\section{Observations and data analysis}

G67.7+1.8 was observed with Chandra in 2007 March 4-5 with the Advanced CCD Imaging Spectrometer (ACIS) using a frame time of $3.2 \mathrm{~s}$. We utilized the whole ACIS-I CCD array to obtain an image of the remnant. The data were reprocessed on 2007 August 7 in order to correct for a systematic aspect offset of $\sim 0.4^{\prime \prime}$, possibly caused by a changing thermal environment ${ }^{1}$. The total observing time on source was $\sim 29 \mathrm{ks}$. Examining the data for times of high background we noticed that the observation was affected by soft-proton flares. Cleaning the data by removing these flares from the data led to an effective exposure time of $\sim 21 \mathrm{ks}$. The analysis was performed in the $0.5-8 \mathrm{keV}$ energy band.

\footnotetext{
${ }^{1}$ See http://cxc.harvard.edu/cal/ASPECT/celmon/ for details.
}

\subsection{Spatial analysis}

A false color image of the ACIS-I data of G67.7+1.8 is displayed in Fig. 2. Comparing the X-ray image with the radio contours obtained from the 1.4 GHz NVSS data (Condon et al. 1998) shows that the X-ray emission is entirely enclosed in the radio arc-like structure (see Fig. 3). The center-filled X-ray emission and the radio shell-like morphology suggest G67.7+1.8 belongs to the category of "mixed-morphology" SNRs (Rho \& Petre 1998).

We have searched for the point sources in the whole ACIS-I data by means of the wavelet source detection algorithm and found 36 sources. These sources are marked by circles in Fig. 2. The limiting count rate of the search was $2 \times 10^{-4} \mathrm{cts} / \mathrm{s}$. The source positions, positional errors, signal-to-noise ratios as well as the estimates of extent of all these 36 sources are given in Table 1. Cross-correlating these sources with the SIMBAD and NED databases did not result in any identification within a search radius of 5 arcsec around each source.

Twelve of these newly detected sources are located within the supernova remnant. Four point-like sources, labeled in Fig. 2 with the numbers \#9, \#10, \#11 and \#35 are located within $\sim 1.5$ arcmin of the geometrical remnant center. Source \#11 is the brightest among them. Hereafter, we designate this source as CXOU195422.97+312902.1. It is also interesting to notice a trail-like radio feature close to the remnant center which has one end apparently coincide with source \#10 (cf. Fig. 3). We designate it as CXOU195424.75+312824.9. This source appears to be 


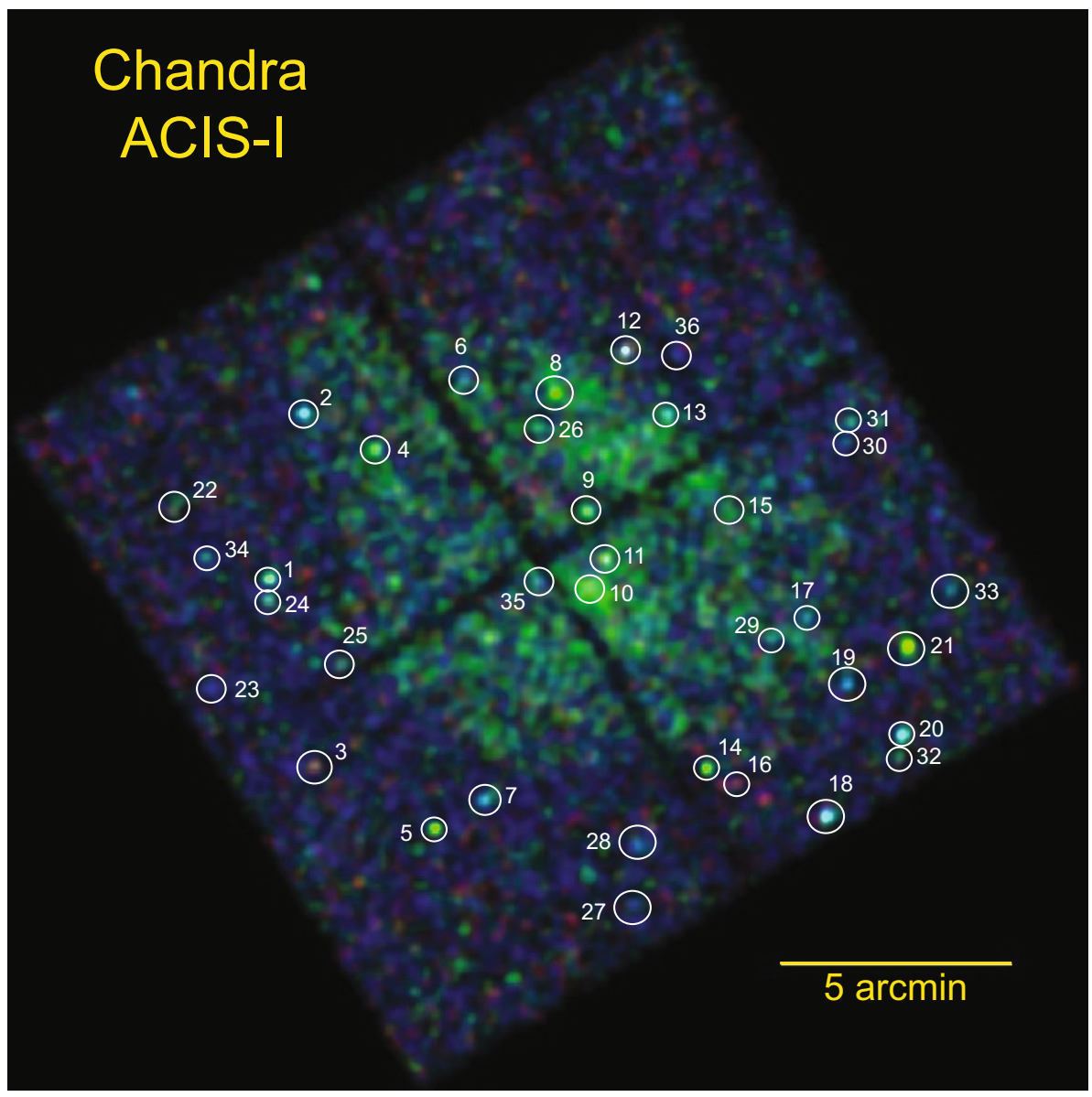

Fig. 2. Chandra ACIS-I false color image of $\mathrm{G} 67.7+1.8$ (red $0.3-0.75 \mathrm{keV}$, green: $0.75-2 \mathrm{keV}$, blue: $2-8 \mathrm{keV}$ ). The binning factor of this image is $2^{\prime \prime}$. It was adaptively smoothed by a Gaussian kernel of $\sigma<6^{\prime \prime}$. 36 point sources are detected in the field-ofview (cf. Table 1). Source \#11 is designated as CXOU195422.97+312902.1, source \#10 is CXOU195424.75+312824.9. Top is north and left is east. slightly extended, which is also suggested by the ratio between its extent and the estimated PSF size (cf. Table 1). However, the low significance level of the detection and the patchy envirnoment of the remnant emission do not allow one to determine if CXOU195424.75+312824.9 is intrinsically extended. In the false colour image, source \#35 appears to have the hardest X-ray emission among these four central point sources (see Fig. 2). We designate this source as CXOU195429.82+312834.1 hereafter.

\subsection{Spectral analysis of $G 67.7+1.8$}

Before we have extracted the remnant spectra, all point-like sources were removed. The spectra were then extracted from the elliptical-shaped regions illustrated in Fig. 4. These regions cover both the northern and the southern radio shells. The background spectra for the corresponding CCD chips were extracted from the boxed regions marked in Fig. 4. We utilized the tool SPECEXTRACT in the data reduction package CIAO 3.4.1 with the calibration data CALDB 3.4.1 to extract the spectra and to compute the response files. After background subtraction there are 1028 and 610 net counts avaliable for the spectral analysis of the northern rim (i.e. the sum of two northern elliptical regions) and southern rim (i.e. the sum of two southern elliptical regions), respectively. The background contributions are found to be $\sim 60 \%$ in both, the northern and southern rims. We binned all the remnant spectra dynamically so as to have at least 50 counts per bin. All the spectral fittings were performed with XSPEC 11.3.2. The parameters of the best-fit models are summarized in Table 2. All quoted errors are $1 \sigma$ for 1 parameter of interest.

\subsubsection{The northern rim}

The X-ray spectrum of the northern rim is shown in Fig. 5 which appears to be rich with line features. We attempted to fit the data with an absorbed collisional ionization equilibrium (CIE) plasma model (XSPEC model: VEQUIL). To examine the abundance of metals, we thawed the parameters individually to see whether the goodness-of-fit can be improved and/or the abundance significantly deviates from the solar value. However, this single component model cannot depict the data beyond $3 \mathrm{keV}$ (with $\chi^{2}=45.32$ for 33 d.o.f.) and requires an unreasonably overabundance of iron and calcium (i.e. hundred times of the solar values).

We proceeded to examine whether the excess in the residual can be modeled by a second thermal component. Adding another CIE component with the solar abundances, we found no improvement in the goodness-of-fit ( $\chi^{2}=47.71$ for 31 d.o.f.).

After examining the residual carefully, we found that combining a CIE model with two additional Gaussian components can model the observed spectrum. The composite model can describe the data within $0.5-8 \mathrm{keV}$ very well: $\chi^{2}=25.41$ for 29 d.o.f. (cf. the lower panel of Fig. 5). All the best-fit parameters are tabulated in Table 2.

The best-fit model yields a hydrogen column density of $n_{\mathrm{H}}=(4.1 \pm 0.9) \times 10^{21} \mathrm{~cm}^{-2}$. Based on the $\mathrm{H} \alpha / \mathrm{H} \beta$ line ratio, Mavromatakis et al. (2001) inferred an optical extinction of $\sim 2$. This value implies a hydrogen column density of $\sim 4 \times 10^{21} \mathrm{~cm}^{-2}$ towards G67.7+1.8 (Predehl \& Schmitt 1995), which is in good agreement with our best-fit value. For comparison, the total galactic neutral hydrogen column density towards $G 67.7+1.8$ is $\sim 10^{22} \mathrm{~cm}^{-2}$ (Kalberla et al. 2005; Dickey \& Lockman 1990). 


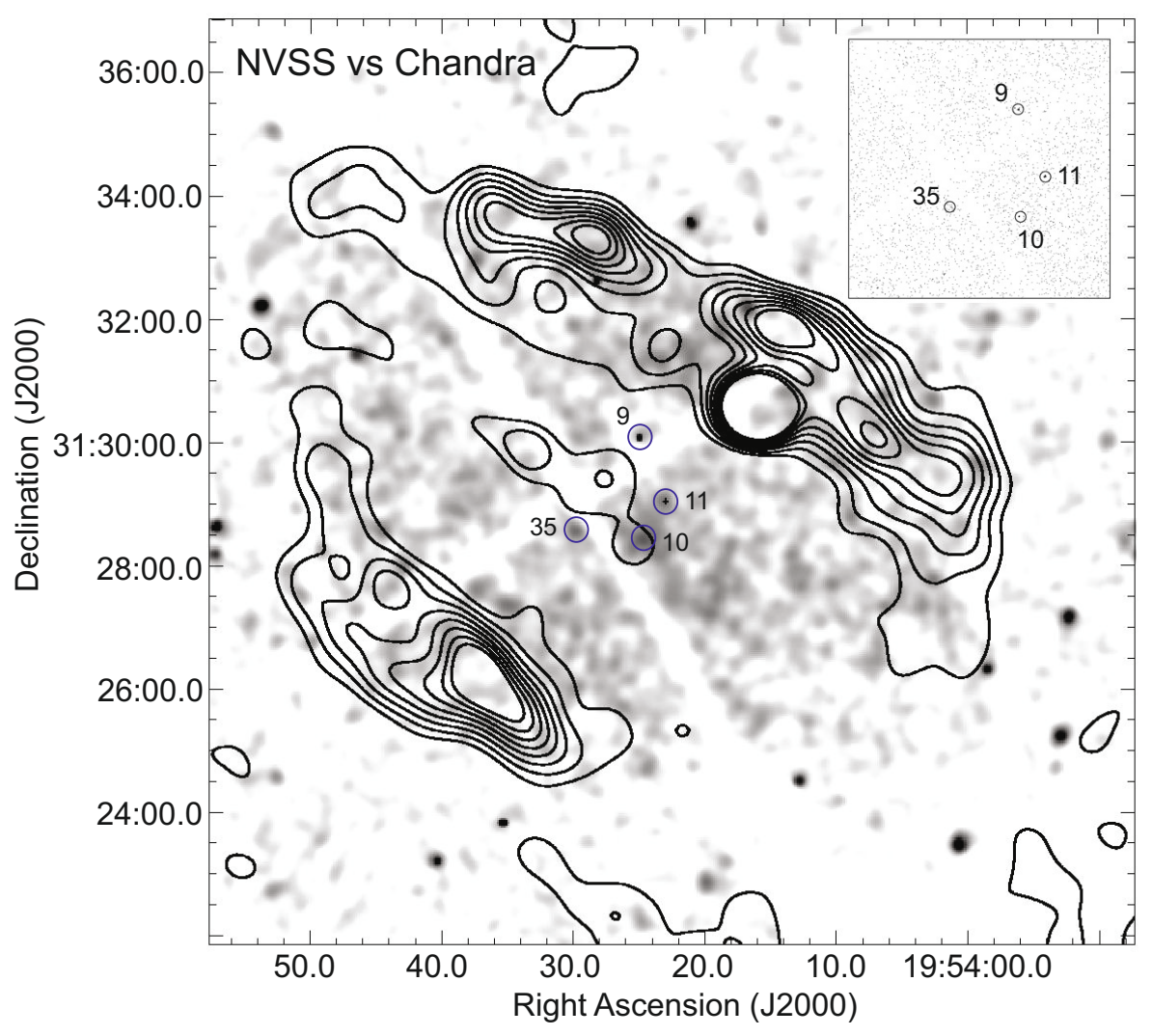

Fig. 3. $15 \times 15$ arcmin field of G67.7+1.8 as observed by Chandra ACIS-I in $0.5-8 \mathrm{keV}$. The radio contours at the levels of $0.6-6 \mathrm{mJy} / \mathrm{beam}$ are from the NVSS data. The blue circles indicate the locations of four point sources near the center of G67.7+1.8. The inset displays the raw image of $4 \times 4$ arcmin of the remnant center with the X-ray sources labeled accordingly.

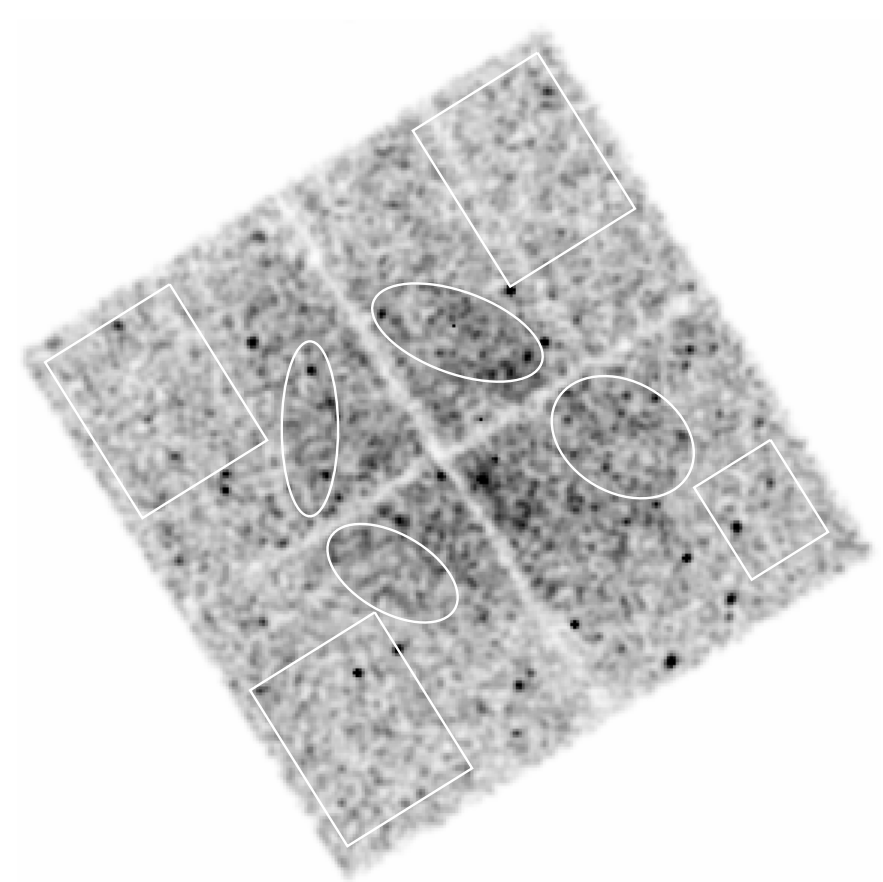

Fig. 4. Illustration of the elliptical-shaped regions used to extract the spectra from the northern and southern rim of G67.7+1.8. The background spectra were extracted from the rectangular regions.

The plasma temperature is found to be $T=6.6_{-0.7}^{+0.5} \times 10^{6} \mathrm{~K}$. For the metal abundance, our analysis suggests that magnesium, silicon and sulphur are overabundant with respect to the solar values $\left(\mathrm{Mg}: 2.6_{-0.7}^{+0.9}, \mathrm{Si}: 2.8_{-1.2}^{+1.5}, \mathrm{~S}: 13.6_{-6.9}^{+8.7}\right)$. The best-fit parameters imply the unabsorbed flux to be $f_{x}=6.7 \times 10^{-13} \mathrm{ergs} \mathrm{cm}^{-2} \mathrm{~s}^{-1}$ in $0.5-8 \mathrm{keV}$.

For the two additional Gaussian line features, the centroids of the line energy were found to locate at $E_{1}=4.0 \pm 0.2 \mathrm{keV}$ and $E_{2}=7.3_{-0.5}^{+3.2} \mathrm{keV}$. The $F W H M \mathrm{~s}$ of the line profiles are $\sigma_{1}=$ $0.3_{-0.1}^{+0.2} \mathrm{keV}$ and $\sigma_{2}=0.9_{-0.3}^{+2.0} \mathrm{keV}$ respectively. The best-fit line fluxes of the features are $f_{\text {line }_{1}}=3.3_{-1.3}^{+1.4} \times 10^{-6}$ photons cm $\mathrm{cm}^{-2} \mathrm{~s}^{-1}$ and $f_{\text {line }_{2}}=2.9_{-1.4}^{+12.7} \times 10^{-5}$ photons $\mathrm{cm}^{-2} \mathrm{~s}^{-1}$ respectively. The possible physical nature and the significance of the emission line features are discussed in Sect. 3.

We have further examined whether a non-equilibrium ionization collisional plasma model (XSPEC model: VNEI) can provide a better fit than a CIE model. With a composite model of VNEI model plus two Gaussian components, We do not found any improvement in the goodness-of-fit $\left(\chi^{2}=25.41\right.$ for 28 d.o.f). All the best-fit parameters are consistent with those inferred from the CIE model. Moreover, the inferred ionization timescale is $\sim 4.5 \times 10^{13} \mathrm{~s} \mathrm{~cm}^{-3}$ which implies the condition of CIE (Borkowski et al. 2001). This justifies the validity of adopting the CIE model in our analysis.

The detection of the emission line features prompted us to investigate their spatial distribution in G67.7 +1.8 by producing an X-ray image in the energy band of 3-8 keV. However, owing to the high background contribution and the relatively limited photon statistics, we cannot identify any significantly excess emission from the background in the image.

\subsubsection{The southern rim}

The X-ray spectrum of the southern rim is shown in Fig. 6. The spectrum appeared to be similar to what we observed from the northern rim, so that we fitted the data again with a CIE model. We have also examined the metal abundances as described in the 
Table 1. Positions of the X-ray sources in the field of ACIS-I as labeled in Fig. 2. The corresponding $1 \sigma$ positional errors, signal-to-noise ratios as well as the estimates of source extent are also tabulated.

\begin{tabular}{|c|c|c|c|c|c|c|}
\hline Source & RA (J2000) & $\operatorname{Dec}(\mathrm{J} 2000)$ & $\delta$ RA & $\delta$ Dec & $S / N^{a}$ & PSFRATIO $^{b}$ \\
\hline & h:m:s & $\mathrm{d}: \mathrm{m}: \mathrm{s}$ & $\operatorname{arcsec}$ & $\operatorname{arcsec}$ & $\sigma_{G}$ & \\
\hline$\overline{1}$ & $19: 54: 57.172$ & $+31: 28: 36.59$ & 0.30 & 0.22 & 13.02 & 1.08 \\
\hline 2 & $19: 54: 53.728$ & $+31: 32: 12.81$ & 0.43 & 0.20 & 14.26 & 1.47 \\
\hline 3 & $19: 54: 52.507$ & $+31: 24: 33.74$ & 0.70 & 0.28 & 4.71 & 0.80 \\
\hline 4 & $19: 54: 46.466$ & $+31: 31: 26.13$ & 0.36 & 0.47 & 6.88 & 2.38 \\
\hline 5 & $19: 54: 40.352$ & $+31: 23: 11.84$ & 0.25 & 0.26 & 10.26 & 0.75 \\
\hline 6 & $19: 54: 37.457$ & $+31: 32: 56.14$ & 0.54 & 0.33 & 4.71 & 2.19 \\
\hline 7 & $19: 54: 35.356$ & $+31: 23: 49.09$ & 0.29 & 0.19 & 9.74 & 0.83 \\
\hline 8 & $19: 54: 28.210$ & $+31: 32: 37.34$ & 0.31 & 0.32 & 5.82 & 2.76 \\
\hline 9 & $19: 54: 24.920$ & $+31: 30: 04.42$ & 0.24 & 0.16 & 10.87 & 5.02 \\
\hline 10 & $19: 54: 24.745$ & $+31: 28: 24.96$ & 0.67 & 0.40 & 3.56 & 9.29 \\
\hline 11 & $19: 54: 22.965$ & $+31: 29: 02.08$ & 0.14 & 0.17 & 21.18 & 6.13 \\
\hline 12 & 19:54:21.016 & $+31: 33: 33.80$ & 0.13 & 0.12 & 24.81 & 1.35 \\
\hline 13 & $19: 54: 16.717$ & $+31: 32: 11.63$ & 0.33 & 0.28 & 6.12 & 2.15 \\
\hline 14 & $19: 54: 12.784$ & $+31: 24: 30.48$ & 0.37 & 0.52 & 8.36 & 1.78 \\
\hline 15 & $19: 54: 10.148$ & $+31: 30: 01.07$ & 0.51 & 0.37 & 2.67 & 1.99 \\
\hline 16 & 19:54:09.741 & $+31: 24: 11.62$ & 0.20 & 0.39 & 3.45 & 0.47 \\
\hline 17 & 19:54:02.592 & $+31: 27: 46.74$ & 0.27 & 0.34 & 4.59 & 1.03 \\
\hline 18 & 19:54:00.649 & $+31: 23: 28.01$ & 0.26 & 0.34 & 16.53 & 0.95 \\
\hline 19 & 19:53:58.504 & $+31: 26: 19.25$ & 0.35 & 0.50 & 9.68 & 1.31 \\
\hline 20 & $19: 53: 52.939$ & $+31: 25: 13.26$ & 0.28 & 0.40 & 15.18 & 0.90 \\
\hline 21 & $19: 53: 52.315$ & $+31: 27: 08.97$ & 0.34 & 0.40 & 13.01 & 1.22 \\
\hline 22 & 19:55:07.125 & $+31: 30: 03.42$ & 0.85 & 0.70 & 2.89 & 0.81 \\
\hline 23 & $19: 55: 03.136$ & $+31: 26: 12.22$ & 0.82 & 0.51 & 3.28 & 0.96 \\
\hline 24 & $19: 54: 57.325$ & $+31: 28: 09.59$ & 0.55 & 0.37 & 6.97 & 1.36 \\
\hline 25 & $19: 54: 49.830$ & $+31: 26: 45.24$ & 0.61 & 0.38 & 3.19 & 1.20 \\
\hline 26 & $19: 54: 30.035$ & $+31: 31: 52.60$ & 0.73 & 0.44 & 4.22 & 5.44 \\
\hline 27 & $19: 54: 20.292$ & $+31: 21: 33.27$ & 0.53 & 0.54 & 4.96 & 0.85 \\
\hline 28 & $19: 54: 19.929$ & $+31: 22: 50.35$ & 0.66 & 0.36 & 4.88 & 1.28 \\
\hline 29 & 19:54:06.334 & $+31: 27: 18.46$ & 0.42 & 0.43 & 4.03 & 1.68 \\
\hline 30 & $19: 53: 58.555$ & $+31: 31: 33.96$ & 0.45 & 0.60 & 4.12 & 1.19 \\
\hline 31 & $19: 53: 58.106$ & $+31: 32: 01.81$ & 0.53 & 0.40 & 4.25 & 1.09 \\
\hline 32 & $19: 53: 53.202$ & $+31: 24: 43.93$ & 0.60 & 0.57 & 4.45 & 0.68 \\
\hline 33 & $19: 53: 47.918$ & $+31: 28: 22.49$ & 0.81 & 0.60 & 3.66 & 1.12 \\
\hline 34 & $19: 55: 03.855$ & $+31: 29: 04.65$ & 0.54 & 0.63 & 4.10 & 0.90 \\
\hline 35 & $19: 54: 29.821$ & $+31: 28: 34.14$ & 0.67 & 0.49 & 3.78 & 10.81 \\
\hline 36 & $19: 54: 15.732$ & $+31: 33: 27.84$ & 0.50 & 0.42 & 4.38 & 1.56 \\
\hline
\end{tabular}

${ }^{a}$ Estimates of source significance in units of Gehrels error: $\sigma_{G}=1+$ $\sqrt{C_{B}+0.75}$ where $C_{B}$ is the background counts; ${ }^{b}$ the ratios between the source extents and the estimates of the PSF sizes.

previous subsection. However, the single component CIE model is not able to describe the data beyond $\sim 4 \mathrm{keV}$ (with $\chi^{2}=38.82$ for 23 d.o.f.). It also requires an unreasonably high abundance of iron.

Inspecting the fit residuals reveals the existence of a Gaussian component centered at $\sim 7 \mathrm{keV}$. However, modeling the data with the line energy of the Gaussian profile as a free parameter did not yield a stable fit. In particular, the centroid of the line cannot be constrained. This can be ascribed to the relatively low photon statistics of the southern rim spectrum. We therefore proceeded to fit the spectrum with the centroid of the line fixed at $7 \mathrm{keV}$. We found that the CIE model complemented with a Gaussian line at $7 \mathrm{keV}$ can describe the data fairly well: $\chi^{2}=31.37$ for 23 d.o.f.. Different from the case of the northern rim, we did not find any excess around $\sim 4 \mathrm{keV}$ in the residual.

The best-fit model yields a hydrogen column density of $n_{\mathrm{H}}=5.9_{-1.1}^{+1.2} \times 10^{21} \mathrm{~cm}^{-2}$ and a plasma temperature of $T=$ $(7.0 \pm 0.7) \times 10^{6} \mathrm{~K}$. Within the $1 \sigma$ errors these values are consistent with those inferred from the spectral fit of the northern rim. The overabundance of magnesium, silicon and sul-
Table 2. Spectral parameters of the best-fit model inferred from the Chandra observed spectra of G67.7+1.8.

\begin{tabular}{|c|c|c|}
\hline & The Northern rim & The Southern rim \\
\hline$n_{\mathrm{H}}\left(10^{21} \mathrm{~cm}^{-2}\right)$ & $4.1 \pm 0.9$ & $5.9_{-1.1}^{+1.2}$ \\
\hline$T\left(10^{6} \mathrm{~K}\right)$ & $6.6_{-0.7}^{+0.5}$ & $7.0 \pm 0.7$ \\
\hline $\mathrm{Mg}$ & $2.6_{-0.7}^{+0.9}$ & $1.7_{-0.7}^{+1.1}$ \\
\hline $\mathrm{Si}$ & $2.8_{-1.2}^{+1.5}$ & $4.7_{-1.7}^{+2.6}$ \\
\hline S & $13.6_{-6.9}^{+8.7}$ & $16.2_{-7.4}^{+11.7}$ \\
\hline $\operatorname{Norm}_{\text {cie }}\left(10^{-4}\right)^{a}$ & $1.4_{-0.4}^{+0.6}$ & $1.0_{-0.4}^{+0.5}$ \\
\hline$E_{1}(\mathrm{keV})$ & $4.0 \pm 0.2$ & - \\
\hline$\sigma_{1}(\mathrm{keV})$ & $0.3_{-0.1}^{+0.2}$ & - \\
\hline $\operatorname{Norm}_{\text {gauss } 1}\left(10^{-6}\right)^{b}$ & $3.3_{-1.3}^{+1.4}$ & - \\
\hline$E_{2}(\mathrm{keV})$ & $7.3_{-0.5}^{+3.2}$ & 7 (fixed) \\
\hline$\sigma_{2}(\mathrm{keV})$ & $0.9_{-0.3}^{+2.0}$ & $1.1_{-0.3}^{+0.4}$ \\
\hline $\operatorname{Norm}_{\text {gauss } 2}^{b}\left(10^{-5}\right)^{b}$ & $2.9_{-1.4}^{+12.7}$ & $1.4 \pm 0.5$ \\
\hline$\chi^{2}$ & 25.41 & 31.37 \\
\hline d.o.f. & 29 & 23 \\
\hline $\begin{array}{l}\text { The normalization } \\
\left.14 / 4 \pi D^{2}\right) \int N_{\mathrm{e}} N_{\mathrm{H}} \mathrm{d} V \\
N_{\mathrm{e}} \text { and } N_{\mathrm{H}} \text { are the } \\
\mathrm{m}^{-3} ; b \text { the normaliz }\end{array}$ & $\begin{array}{l}\text { of CIE mod } \\
\text { where } D \text { is the } \\
\text { ost-shock electron } \\
\text { tion of the Gauss }\end{array}$ & $\begin{array}{l}\text { is expressed } \\
\text { ource distance i } \\
\text { and hydrogen der } \\
\text { an model is in u }\end{array}$ \\
\hline
\end{tabular}

Table 3. Spectral characteristics of the central X-ray sources within the supernova remnant G67.7+1.8.

\begin{tabular}{cccc}
\hline \hline Source & $\begin{array}{c}\text { Net rate } \\
\left(10^{-3} \mathrm{cts} / \mathrm{s}\right)\end{array}$ & $\Gamma$ & $\begin{array}{c}f_{x} \\
\left(\mathrm{ergs} \mathrm{cm}^{-2} \mathrm{~s}^{-1}\right)\end{array}$ \\
\hline 9 & $1.17 \pm 0.24$ & $3.2_{-0.8}^{+1.0}$ & $2.8 \times 10^{-14}$ \\
10 & $0.42 \pm 0.26$ & $2.7_{-1.7}^{+2.4}$ & $9.8 \times 10^{-15}$ \\
11 & $2.54 \pm 0.39$ & $2.7 \pm 0.4$ & $4.5 \times 10^{-14}$ \\
35 & $0.75 \pm 0.23$ & $1.3_{-0.5}^{+0.7}$ & $1.2 \times 10^{-14}$ \\
\hline
\end{tabular}

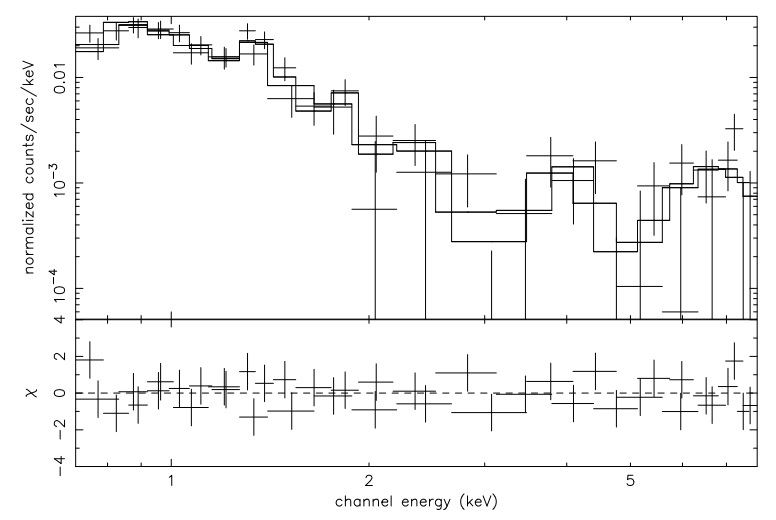

Fig. 5. X-ray spectrum of the emission from the northern rim (upper panel) observed by Chandra's ACIS-I2 and I3 CCDs. The residuals are from fitting an absorbed collisional ionization equilibrium plasma model plus two additional Gaussian components (lower panel).

phur with respect to the solar values are $\mathrm{Mg}: 1.7_{-0.7}^{+1.1}, \mathrm{Si}: 4.7_{-1.7}^{+2.6}$, $\mathrm{S}: 16.2_{-7.4}^{+11.7}$. The best-fit parameters imply the unabsorbed flux to be $f_{\text {plasma }}=4.2 \times 10^{-13} \mathrm{ergs} \mathrm{cm}^{-2} \mathrm{~s}^{-1}$ in $0.5-8 \mathrm{keV}$.

For the Gaussian line feature at $7 \mathrm{keV}$, the $F W H M$ of the profile is found to be $1.1_{-0.3}^{+0.4} \mathrm{keV}$. The line flux of this feature is $f_{\text {line }}=(1.4 \pm 0.5) \times 10^{-5}$ photons $\mathrm{cm}^{-2} \mathrm{~s}^{-1}$. 


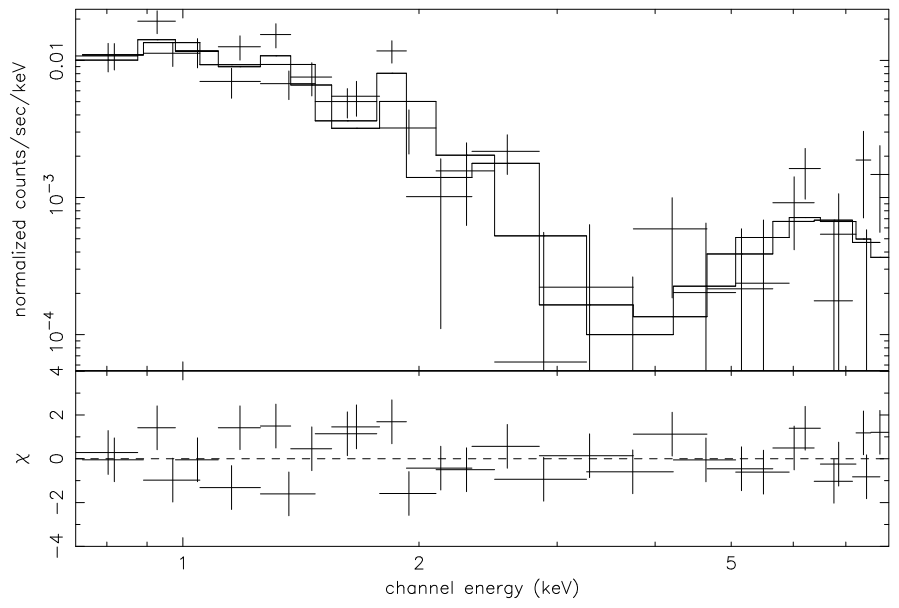

Fig. 6. X-ray spectrum of the emission from the southern rim (upper panel) observed by Chandra's ACIS-I0 and I1 CCDs. The residuals are from fitting an absorbed collisional ionization equilibrium plasma model plus an additional Gaussian component with the centroid fixed at $7 \mathrm{keV}$ (lower panel).

Adopting the VNEI model does not provide a better description of the data than the CIE model. The composite model of VNEI component plus the Gaussian fixed at $7 \mathrm{keV}$ results in a goodness-of-fit of $\chi^{2}=31.36$ for 22 d.o.f.. All the best-fit parameters are consistent with those inferred from the CIE model. The ionization timescale is found to be $\sim 4.5 \times 10^{13} \mathrm{~s} \mathrm{~cm}^{-3}$ which is similar to that inferred from the northern rim. Therefore, the CIE condition is justified and we will not further consider the VNEI model.

\section{Spectral analysis of the central point sources}

CXOU195422.97+312902.1 (source \#11) is the brightest one among the four point sources near to the remnant center. We extracted its spectrum from a circular region with a radius of 10 arcsec centered at the source position (cf. Table 1). The background spectrum was extracted from a nearby region from a circle of 10 arcsec radius centered at RA $=19^{\mathrm{h}} 54^{\mathrm{m}} 23.234^{\mathrm{s}}$, Dec $=31^{\circ} 29^{\prime} 22.75^{\prime \prime}(\mathrm{J} 2000)$. After background subtraction, 54 net counts were avaliable for the spectral analysis of the source. Since the location of CXOU195422.97+312902.1 is close to the edge of ACIS-I3 CCD, we computed the response files manually with the CIAO tools MKARF and MKRMF. The spectrum was binned dynamically so as to have at least 5 counts per bin. In view of the small photon statistic of these sources, we adopted the $C$-statistic (Cash 1979) for all the fittings. To better constrain the parameters, we fixed the hydrogen column density at the optical extinction inferred value (i.e. $4 \times 10^{21} \mathrm{~cm}^{-2}$ ).

Fitting with a power-law model results in a reasonable fit that yields a photon index of $\Gamma=2.7 \pm 0.4$ and a normalization at $1 \mathrm{keV}$ of $1.4_{-0.3}^{+0.4} \times 10^{-5}$ photons $\mathrm{keV}^{-1} \mathrm{~cm}^{-2} \mathrm{~s}^{-1}$. The best-fit column density is consistent with the values inferred from the spectra of the supernova remnant emission within the $1 \sigma$ error bound. The unabsorbed flux deduced for the best-fit power-law model parameters is $f_{x}=4.5 \times 10^{-14} \mathrm{ergs} \mathrm{cm}^{-2} \mathrm{~s}^{-1}$ in $0.5-8 \mathrm{keV}$.

A blackbody model can describe the spectrum of this source equally well. The best-fit model implies a temperature of $T=$ $4.6_{-0.6}^{+0.7} \times 10^{6} \mathrm{~K}$. The radius of the projected blackbody emitting area is in the range $\sim 170-310 \mathrm{~m}$ and $\sim 410-750 \mathrm{~m}$ for the adopted distances of $7 \mathrm{kpc}$ and $17 \mathrm{kpc}$, respectively (see Sect. 3 for the discussion of the remnant distance). The unab- sorbed flux deduced for the best-fit blackbody model is $f_{x}=$ $2.6 \times 10^{-14} \mathrm{ergs} \mathrm{cm}^{-2} \mathrm{~s}^{-1}$ in $0.5-8 \mathrm{keV}$.

For the other three fainter central X-ray sources it is interesting to compare their brightness and hardness with those of CXOU195422.97+312902.1. To do so, we have prepared their spectra and the response files in the same way as we did for CXOU195422.97+312902.1. Fixing the column density at $n_{\mathrm{H}}=4 \times 10^{21} \mathrm{~cm}^{-2}$ we obtained the photon indices by fitting a power-law model to their spectra. The fitted parameters are summarised in Table 3. The photon index provides a measure of the hardness of these X-ray sources. Whereas sources \#9 and CXOU195424.75+312824.9 (source \#10) are as soft as CXOU195422.97+312902.1, CXOU195429.82+312834.1 (source \#35) appears to show harder X-ray emission. We have also computed the absorption-corrected fluxes from the inferred power-law parameters which are given in Table 3 .

\subsection{Optical identifications of the central point sources}

Given the limited photon statistics of the central point sources, the spectral analysis is not very constraining. Even for the brightest object CXOU195422.97+312902.1, we cannot distinguish its emission nature between the thermal and the non-thermal scenarios. To investigate if these sources are promising neutron star candidates we proceeded to search for their optical counterparts by utilizing the USNO-B1.0 catalogue (Monet et al. 2003).

For the search regions, we combine the positional errors of each source with the pointing uncertainty of the spacecraft. The uncertainty can be estimated from the distribution of aspect offset for a sample of point sources with accurately known celestial positions $^{2}$. There is $68 \%$ of 70 sources imaged on ACIS-I have offsets smaller than $\sim 0.4$ arcsec. We adopted this value as the astrometric uncertainty and added to the quoted positional errors (cf. Table 1) in quadrature for each coordinate.

For source \#9, we have identified an optical counterpart at 0.39 arcsec away from its X-ray position. It has a magnitude of $B=15.93$ and $R=14.69$ which implies an X-ray-to-optical flux ratio to be $f_{X} / f_{\text {opt }} \sim 10^{-3}$. This ratio suggests source \#9 is likely to be a field star which typically has a ratio $f_{X} / f_{\text {opt }}<0.3$ (Maccacaro et al. 1988).

For CXOU195422.97+312902.1, an optical counterpart with $B=15.92$ and $R=14.43$ is consistent with its X-ray position. For this source the X-ray-to-optical flux ratio is at the level of $\sim 10^{-3}$ which is in agreement with what is expected for a field star.

We do not find any optical counterpart for CXOU195424.75+312824.9 and CXOU195429.82+312834.1. Adopting the limiting magnitude in the USNO-B1.0 catalogue to be 21 (cf. Monet et al. 2003), the upper limit of $f_{X} / f_{\text {opt }}$ is found to be $>0.1$. This upper limit is not particular constraining. A deep optical observation is required to differentiate the nature of these two sources from the star with $f_{X} / f_{\text {opt }}<0.3$ and active galactic nuclei with $f_{X} / f_{\text {opt }}<50$ (Maccacaro et al. 1988; Stocke et al. 1991).

\section{Summary and discussion}

We have performed a detailed spectro-imaging X-ray study of the supernova remnant G67.7+1.8 with Chandra. Various properties of G67.7+1.8, including distance, age and explosion energy, are still poorly constrained. The type of the supernova and the nature of the progenitor are also unknown. With the

2 http://cxc.harvard.edu/cal/ASPECT/celmon/ 
Chandra observation it became possible for the first time to shed some more detailed light on the X-ray emission nature of G67.7+1.8 and its central point sources.

The spectra of the remnants northern and southern rims can be described with a single temperature CIE model plus additional Gaussian components. Our analysis suggests a plasma temperature of $\sim 7 \times 10^{6} \mathrm{~K}$. Assuming that the ions and electrons are fully equilibrated in the shock, the temperature implies that the blast wave has a velocity of $\sim 800 \mathrm{~km} \mathrm{~s}^{-1}$ which is at the same order of magnitude as inferred from the RASS data (Mavromatakis et al. 2001). Although the single temperature plasma model describes our observed spectra very well, most supernova remnants require multi-temperature models to depict their X-ray spectra (e.g. Gaensler et al. 2008). A composite model can describe the shocked ejecta and the swept-up material from the surrounding with the components of high temperature and low temperature, respectively. However, for a more detailed modeling of the remnant spectrum, data with improved photon statistics are badly needed.

Utilizing the $\Sigma-D$ relation, Case \& Bhattacharya (1998) estimated the distance to G67.7+1.8 to be $\sim 17 \mathrm{kpc}$. However, the large uncertainties on the proportionality factor and the exponent of the $\Sigma-D$ relation make the distance poorly constrained and results in a range of 7-27 kpc. On the other hand, the observation of the optical filaments suggests a distance less than $17 \mathrm{kpc}$, otherwise the detection of the optical radiation would be very difficult to explain in view of the measured interstellar extinction (Mavromatakis et al. 2001). In the following calculations we assume the distance to be in the range of $7-17 \mathrm{kpc}$.

The emission measure inferred from the spectral fits of the remnant emission allows us to estimate the hydrogen density, $N_{\mathrm{H}}$, in the shocked regions. Assuming the post-shock densities of hydrogen and electrons are uniform in the extraction region, the normalization of the CIE model can be approximated by $N_{\text {cie }} \simeq 10^{-14} N_{\mathrm{H}} N_{\mathrm{e}} V / 4 \pi D^{2}$, where $D$ is the distance to G67.7+1.8 in $\mathrm{cm}$ and $V$ is the volume of interest in units of $\mathrm{cm}^{3}$. Assuming a geometry of oblated spheroid, the volume of interest for the northern rim and the southern rim are $3.9 \times 10^{55} \mathrm{~d}_{\mathrm{kpc}}^{3} \mathrm{~cm}^{3}$ and $2.6 \times 10^{55} \mathrm{~d}_{\mathrm{kpc}}^{3} \mathrm{~cm}^{3}$, respectively. Here $d_{\mathrm{kpc}}$ is the remnant distance in units of kpc. Assuming a fully ionized plasma, $N_{\mathrm{H}} N_{\mathrm{e}}$ can be approximated by $0.7 N_{\mathrm{H}}^{2}$. The best-fit normalizations imply a post-shock hydrogen density of $N_{\mathrm{H}} \sim 0.06-0.1 \mathrm{~cm}^{-3}$ for the assumed range of distances.

Assuming G67.7+1.8 is in a Sedov phase, the shock temperature can be estimated by:

$T_{\mathrm{s}} \simeq 8.1 \times 10^{6}\left(\frac{E_{51}}{N_{\mathrm{H}_{-1}}}\right)^{\frac{2}{5}} t_{4}^{-\frac{6}{5}} \mathrm{~K}$

where $t_{4}, E_{51}$ and $N_{\mathrm{H}_{-1}}$ are the time after the explosion in units of $10^{4}$ years, the released kinetic energy in units of $10^{51}$ ergs and the post-shock hydrogen density of $0.1 \mathrm{~cm}^{-3}$ respectively (McCray 1987). Mavromatakis et al. (2001) has argued the explosion energy should be significantly less than the canonical value of $10^{51}$ ergs. Adopting an explosion energy of $E=$ $10^{50}$ ergs and the plasma temperature inferred from our spectral analysis as an estimate of $T_{\mathrm{s}}$ (i.e. $\sim 7 \times 10^{6} \mathrm{~K}$ ), the range of $N_{\mathrm{H}} \sim 0.06-0.1 \mathrm{~cm}^{-3}$ yields the age of $667.7+1.8$ to be $\sim 5000-6000$ yrs. On the other hand, an age bracket of $\sim 11000-$ $13000 \mathrm{yrs}$ is suggested from $E=10^{51} \mathrm{ergs}$.
Taking the estimates of the age, explosion energy and the density of the interstellar medium, we calculate the theoretical size of the remnant by (Culhane 1977):

$R_{\mathrm{s}} \simeq 21.5\left(\frac{E_{51}}{N_{\mathrm{H}_{-1}}}\right)^{\frac{1}{5}} t_{4}^{\frac{2}{5}} \mathrm{pc}$.

From the X-ray image, we estimated the angular radius of the remnant to be $\theta_{\mathrm{s}} \simeq 5 \operatorname{arcmin}$. Comparing $R_{\mathrm{s}}$ and $\theta_{\mathrm{s}}$, we can estimate the distance by $d=R_{\mathrm{S}} / \theta_{\mathrm{s}}$. For an explosion energy of $E=10^{50} \mathrm{ergs}$, the distance is estimated to be in a range of $\sim 7-$ $12 \mathrm{kpc}$ for $N_{\mathrm{H}}=0.06-0.1 \mathrm{~cm}^{-3}$ and $t=5000-13000 \mathrm{yrs}$. On the other hand, if $E=10^{51} \mathrm{ergs}$ is adopted, it implies a distance bracket of $\sim 12-20 \mathrm{kpc}$ for the same ranges of $N_{\mathrm{H}}$ and $t$ in the previous calculation.

As aforementioned, the optical extinction suggests that the distance is at the lower side. On the basis of our estimation, for a preferable distance much smaller than $17 \mathrm{kpc}$, we favor a scenario that the explosion is less energetic than a canonical supernova (i.e. $E<10^{51} \mathrm{ergs}$ ), which is consistent with the conclusion drawn by Mavromatakis et al. (2001) from their optical study.

Assuming that the remnant G67.7+1.8 is the result of a low energy supernova explosion has interesting implications for the properties of its progenitor. In the context of the current understanding in stellar evolution, a supernova with kinetic energy of $\sim 10^{50}$ ergs can be a result of two different evolutionary tracks.

In the first scenario, a oxygen-neon-magnesium (ONeMg) core can be formed before Neon and subsequent nuclear burning take place. If the neutrino cooling is efficient enough, the core temperature will be reduced and prevents further nuclear fusion. When the ONeMg core reaches the Chandrasakar mass, the electron degenerate pressure is no longer able to support the core. Furthermore, electron capture by ${ }^{24} \mathrm{Mg}$ and ${ }^{20} \mathrm{Ne}$ will further reduce the electron degenerate pressure in the core and trigger the core to collapse (Miyaji et al. 1980; Gutiérrez et al. 2005). This is known as the electron-capture supernova. Most simulations have shown that this type of explosion has a rather low energy (see Eldridge et al. 2007). The progenitor's mass of an electron-capture supernova is limited in a narrow range of $\sim 6-8 M_{\odot}$ (Eldridge et al. 2007). For more massive stars, they will go through all stages to silicon burning. In less massive stars, ONeMg cores cannot be formed.

There is another possibility to produce a low energy supernova. The exact evolution of a collapsing star after neutrino reheating depends on the rate of early infall of stellar material on the collapsed core and on the binding energy of the envelope. If both are large, which is the case in high-mass stars, the energy required to accelerate and heat up the ejecta is not available, preventing a successful explosion and resulting in a supernova which appears to be under-energetic (see Heger et al. 2003; Eldridge \& Tout 2004). The progenitor of this evolutionary track is likely to be $\gtrsim 20 M_{\odot}$.

We have also discovered emission line features in the remnant spectrum. The feature at $E=4.0 \pm 0.2 \mathrm{keV}$ is detected only in the northern rim. The width of this feature $(\sigma=0.3 \mathrm{keV})$ is a little larger than the energy resolution of ACIS-I at $4 \mathrm{keV}$ (i.e. $\sigma \sim 0.1 \mathrm{keV}$ ). This might indicate that the feature can be a blend of several lines. Its energy centroid is close to a number of $\mathrm{K}$ emission lines from ${ }^{44} \mathrm{Ca}$ and/or ${ }^{44} \mathrm{Sc}$ (cf. Table 5 in Iyudin et al. 2005 and references therein). Therefore, we speculate that the feature at $4 \mathrm{keV}$ can possibly comprises these lines.

If the identification is correct, it suggests a possible presence of ${ }^{44} \mathrm{Ti}$ because both ${ }^{44} \mathrm{Ca}$ and ${ }^{44} \mathrm{Sc}$ are produced in the decay chain ${ }^{44} \mathrm{Ti} \rightarrow{ }^{44} \mathrm{Sc} \rightarrow{ }^{44} \mathrm{Ca}$. The half-life of ${ }^{44} \mathrm{Ti}$ is $\sim 60 \mathrm{yrs}$. If the 
line feature at $4 \mathrm{keV}$ is indeed from the decay of ${ }^{44} \mathrm{Ti}$, its short half-life implies that the remnant should be rather young. Also, the production of ${ }^{44} \mathrm{Ti}$ in the supernova is very sensitive to the explosion mechanism and the ejecta dynamics (see discussion in Iyudin et al. 2005). Therefore, obtaining the yield and spatial distribution of ${ }^{44} \mathrm{Ti}$ can help to further constrain the physical properties of the remnant as well as the nature of the progenitor.

On the other hand, the broadening of the feature can also result from the motion of the ejecta. However, this would imply the ejecta to have a velocity of $\sim 10000 \mathrm{~km} \mathrm{~s}^{-1}$. This is not consistent with $\mathrm{G} 67.7+1.8$ being the result of a low energy supernova explosion as inferred from our X-ray data analysis as well from optical observation. Furthermore, the limited energy resolution of imaging data precludes any accurate measurement of the line width. High resolution grating spectroscopy is required to disentangle the possible blend of lines as well as determine their widths.

Apart from the feature at $4 \mathrm{keV}$, we have also found an emission line feature at $\sim 7 \mathrm{keV}$ in both, the northern and southern rims. The large errors of the energy centroid and the width do not allow a firm identification. A deep observation is required to better constrain these parameters. We note that the approximate energy of the feature is rather close to the $\mathrm{Fe}-\mathrm{K}$ emission. This leads us to speculate that the feature can be a result from unresolved $\mathrm{Fe} \mathrm{K}_{\alpha}$ and $\mathrm{K}_{\beta}$ lines. This also requires data with high spectroscopic resolution for further investigation.

The Chandra observation of G67.7+1.8 also leads to the detection of X-ray point sources at the proximity of the remnant's geometrical center. However, the limited photon statistics do not allow a constraining spectral analysis of these sources. The non-detection of optical counterparts for CXOU195424.75+312824.9 and CXOU195429.82+312834.1 leaves these two objects as possible candiates for a stellar compact remnant. A dedicated deep optical observation is desirable to better constraining the nature of the point sources.

Acknowledgements. C.Y.H. is supported by the Croucher Foundation Postdoctoral Fellowship. C.Y.H. would like to thank Herbert H. B. Lau and J. J. Eldridge for discussing the possible progenitors of low energy supernova.
The authors would also like to thank the referee for providing many useful suggestions to improve the quality of the manuscript.

\section{References}

Becker, W., Hui, C. Y., Aschenbach, B., \& Iyudin, A. 2006, A\&A, submitted [arXiv: astro-ph/0607081]

Becker, W. 2008, in Neutron Stars and Pulsars, ed. W. Becker, Astrophysics and Space Science Library (Springer), 99

Borkowski, K. J., Lyerly, W. J., \& Reynolds, S. P. 2001, ApJ. 548. 820

Case, G. L., \& Bhattacharya, D. 1998, ApJ, 504, 761

Cash, W. 1979, ApJ, 228, 939

Condon, J. J., Cotton, W. D., \& Greisen, E. W. 1998, AJ, 115, 1693

Culhane, J. L. 1977, in Supernovae, D. Reidel Publishing Co., ed. D. N. Schram (Dordrecht: Holland), 29

De Luca, A., Caraveo, P. A., \& Mereghetti, S. 2006, Science, 313, 814

Dickey, J. M., \& Lockman, F. J. 1990, ARA\&A, 28, 215

Eldridge, J. J., \& Tout, C. A. 2004, MNRAS, 353, 87

Eldridge, J. J., Mattila, S., \& Smartt, S. J. 2007, MNRAS, 376, L52

Gaensler, B. M., Tanna, A., Slane, P. O., et al. 2008, ApJ, 680, L37

Green D. A. 2006, A Catalogue of Galactic Supernova Remnants (April version), Mullard Radio Astronomy Observatory, Cavendish Laboratory (UK: Cambridge)

Gutiérrez, J., Canal, R., \& García-Berro, E. 2005, A\&A, 435, 231

Halpern, J. P., Gotthelf, E. V., Camilo, F., \& Seward, F. D. 2007, ApJ, 665, 1304

Heger, A., Fryer, C. L., Woosley, S. E., Langer, N., \& Hartmann, D. H. 2003, ApJ, 591, 288

Hui, C. Y., \& Becker, W. 2006, A\&A, 454, 543

Iyudin, A. F., Aschenbach, B., Becker, W., Dennerl, K., \& Haberl, F. 2005, A\&A, 429,225

Kalberla, P. M. W., Burton, W. B., Hartmann, D., et al. 2005, A\&A, 440, 775

Li, X.-D., 2007, ApJ, 666, L81

Lorimer, D. R., Lyne, A. G., \& Camilo, F. 1998, A\&A, 331, 1002

Maccacaro, T., Gioia, I. M., Wolter, A., Zamorani, G., \& Stocke, J. T. 1988, ApJ, 326,680

Mavromatakis, F., Papamastorakis, J., Ventura, J., et al. 2001, A\&A, 370, 265

McCray, R. 1987, in Spectroscopy of Astrophysical Plasmas, ed. Davis \& Smith, 248

Miyaji, S., Nomoto, K., Yokoi, K., \& Sugimoto, D. 1980, PASJ, 32, 303

Monet, D. G., Levine, S. E., Canzian, B., et al. 2003, AJ, 125, 984

Predehl, P., \& Schmitt, J. H. M. M. 1995, A\&A, 293, 889

Rho, J., \& Petre, R. 1998, ApJ, 503, L167

Stocke, J. T., Morris, S. L., Gioia, I. M., et al. 1991, ApJS, 76, 813

Taylor, A. R., Wallace, B. J., \& Goss, W. M. 1992, AJ, 103, 931

Vink, J., Laming, J. M., Kaastra, J. S., et al. 2001, ApJ, 560, L79 